\title{
Serum Bicarbonate and Structural and Functional Cardiac Abnormalities in Chronic Kidney Disease - A Report from the Chronic Renal Insufficiency Cohort Study
}

\author{
Mirela Dobre $^{\mathrm{a}}$ Jason Roy ${ }^{\mathrm{c}}$ Kaixiang Tao ${ }^{c}$ Amanda H. Anderson ${ }^{c}$ \\ Nisha Bansal ${ }^{e}$ Jing Chen ${ }^{f}$ Rajat Deo ${ }^{d}$ Paul Drawz $^{g}$ Harold I. Feldman $^{c}$ \\ L. Lee Hamm ${ }^{f}$ Thomas Hostetter ${ }^{a}$ John W. Kusek ${ }^{\text {h }}$ Claudia Lora ${ }^{i}$ \\ Akinlolu O. Ojo Kumar Shramak Mahboob Rahman ${ }^{a, b}$ the CRIC Study \\ Investigators

\begin{abstract}
aDivision of Nephrology and Hypertension, University Hospital Case Medical Center, Case Western Reserve University, and ${ }^{b}$ Louis Stokes Cleveland VA Medical Center, Cleveland, Ohio., ${ }^{c}$ Center for Clinical Epidemiology Philadelphia, Pa., 'Division of Nephrology, Kidney Research Institute, University of Washington, Seattle, Wash., fSection of Nephrology and Hypertension, Department of Medicine, Tulane University School of Medicine, New Orleans, La., 9 Division of Renal Diseases and Hypertension, University of Minnesota, Minneapolis, Minn., ${ }^{h}$ NIDDK, National Institutes of Health, National Institute of Diabetes and Digestive and Kidney Diseases, 'Department of Medicine, Division of Nephrology, University of Illinois at Chicago, Chicago, III., 'j Department of Medicine - Nephrology, University of Michigan, Ann Arbor, Mich., and kCenter for Renal Translational Medicine, Division of Nephrology-Hypertension, University of California at San Diego, San Diego, Calif., USA
\end{abstract} \\ and Biostatistics and ${ }^{\mathrm{d} C a r d i o v a s c u l a r}$ Medicine Division, University of Pennsylvania Perelman School of Medicine,
}

\section{Key Words}

Serum bicarbonate - Left ventricular hypertrophy · Left ventricular geometry · Chronic kidney disease

\begin{abstract}
Background: Heart failure (HF) is a frequent occurrence in chronic kidney disease (CKD) patients and predicts poor survival. Serum bicarbonate is associated with increased rates of HF in CKD; however, the mechanisms leading to this association are incompletely understood. This study aims to assess whether serum bicarbonate is independently associated with structural and functional cardiac abnormalities in CKD. Methods: The association between serum bicarbonate
\end{abstract}

\section{KARGER}

E-Mail karger@karger.com www.karger.com/ajn and left ventricular (LV) hypertrophy (LVH), LV mass indexed to height ${ }^{2.7}$, LV geometry, ejection fraction (EF) and diastolic dysfunction was assessed in 3,483 participants without NYHA class III/IV HF, enrolled in the Chronic Renal Insufficiency Cohort study. Results: The mean estimated glomerular filtration rate was $42.5 \pm 17 \mathrm{ml} / \mathrm{min} / 1.73 \mathrm{~m}^{2}$. The overall prevalence of LVH was $51.2 \%$, with $57.8,50.9$ and $47.7 \%$ for bicarbonate categories $<22,22-26$ and $>26 \mathrm{mmol} / \mathrm{l}$, respectively. Participants with low bicarbonate were more likely to have LVH and abnormal LV geometry (OR 1.32; 95\% Cl 1.071.64, and OR 1.57; 95\% Cl 1.14-2.16, respectively). However,

CRIC Study Investigators are listed in the Appendix.
(C) 2016 Published by S. Karger AG, Basel 0250-8095/16/0436-0411\$39.50/0
Mirela Dobre, MD

Division of Nephrology and Hypertension

University Hospital Case Medical Center, Case Western Reserve University 11100 Euclid Avenue, Cleveland, OH 44106 (USA)

E-Mail Mirela.Dobre@uhhospitals.org 
the association was not statistically significant after adjustment for demographics, traditional cardiovascular risk factors, medications and kidney function (OR 1.07; 95\% Cl 0.661.72 , and OR $1.27 ; 95 \% \mathrm{Cl} 0.64-2.51$, respectively). No association was found between bicarbonate and systolic or diastolic dysfunction. During follow-up, no significant changes in LV mass or EF were observed in any bicarbonate strata. Conclusions: In a large CKD study, serum bicarbonate was associated with LV mass and concentric LVH; however, this association was attenuated after adjustment for clinical factors suggesting that the observed cardiac effects are mediated through yet unknown mechanisms.

() 2016 Published by S. Karger AG, Basel

\section{Introduction}

Acid-base disturbances are common in patients with chronic kidney disease (CKD) [1]. The kidneys maintain the acid-base equilibrium by excreting acid in amounts that are equal to the extra-renal acid production by reclaiming filtered bicarbonate and regenerating base through the excretion of ammonium and titratable acid [2]. A decline in kidney function leads to impaired generation of new bicarbonate and abnormal or preserved tubular bicarbonate reabsorption resulting in a low-degree chronic metabolic acidosis commonly observed in CKD patients $[3,4]$.

Heart failure (HF) is common and associated with high morbidity and mortality in patients with CKD [5]. Also, left ventricular (LV) hypertrophy (LVH) is common in CKD [6-8] and is an important predictor of long-term adverse outcomes $[9,10]$. The role of acid-base abnormalities on cardiac muscle is not completely understood. In animal models, mild renal insufficiency results in early cardiac fibrosis and impaired diastolic function, which progresses to more global LV remodeling and dysfunction [11]. Acutely induced myocardial acidosis adversely affects the recovery of micro-vascular and LV function and increases indices of apoptosis with potential longterm effect on cardiac performance [12]. A low $\mathrm{pH}$ has been associated with the reduction of $\mathrm{Na}+-\mathrm{K}+$-ATPase activity in myocardial cells [13], which could lead to reduced myocardial contractility and congestive HF [14]. Whether the low-grade chronic metabolic acidosis associated with CKD plays a role in $\mathrm{LV}$ remodeling is unknown.

Our previous work in the Chronic Renal Insufficiency Cohort (CRIC) study showed a 'U-shaped' association between serum bicarbonate and clinical HF events, with an increased risk of HF at both extremes of serum bicar- bonate $[15,16]$. In another study, the magnitude of change in serum bicarbonate level, regardless of direction, was correlated with increased mortality in a cohort of patients hospitalized for HF [17]. Whether serum bicarbonate levels are associated with cardiac structural or functional abnormalities that predispose to these clinical outcomes is not known.

The goal of this analysis was to evaluate the association between serum bicarbonate levels and LV structure and function, including LV mass and hypertrophy, ejection fraction (EF), measures of diastolic dysfunction and LV geometry in a cohort of patients with CKD.

\section{Methods}

\section{Study Design and Population}

The CRIC study enrolled 3,943 individuals aged 21-74 with estimated glomerular filtration rate (eGFR) $20-70 \mathrm{ml} / \mathrm{min} / 1.73 \mathrm{~m}^{2}$, from June 2003 to December 2008 at 7 clinical centers across the United States (Ann Arbor, Mich.; Baltimore, Md.; Chicago, Ill.; Cleveland, Ohio.; New Orleans, La.; Philadelphia, Pa.; and Oakland, Calif.). Study design and baseline participant characteristics have been previously published [18-20]. Major exclusion criteria included prior dialysis treatment lasting $>1$ month, NYHA Class III/IV $\mathrm{HF}$, polycystic kidney disease, or other primary renal diseases requiring active immunosuppression, human immunodeficiency virus infection, pregnancy, and inability to give informed consent or institutionalization. Participants underwent annual study visits and telephone follow-up twice a year. Echocardiogram evaluation was performed at study years 1, 4 and 7 . The study population for this analysis included 3,483 participants after exclusion of 37 with missing serum bicarbonate measurements at study year 1 . There was no statistically significant difference between study participants and those excluded from the analyses (online suppl. fig. 1S; for all online suppl. material, see www.karger.com/doi/10.1159/000446860). Study participants provided written informed consent and are followed annually under protocols approved by Institutional Review Boards at each of the CRIC study clinical centers.

\section{Data Collection}

Main Predictor

Serum bicarbonate was measured annually using an enzymatic procedure with phosphoenolpyruvate carboxylase on the Ortho Vitros platform at the University of Pennsylvania Core Laboratory. The current analysis examined bicarbonate continuously per $1 \mathrm{mmol} / \mathrm{l}$ increase, and categorically using the following groups of serum bicarbonate: $<22,22-26$ (reference group) and $>26 \mathrm{mmol} / \mathrm{l}$. The cut points for categories of serum bicarbonate used are based on the current recommended clinical guidelines in CKD and available literature [15].

\section{Outcomes}

The study outcomes were 2D mode echocardiography measures of cardiac structure and function. All echocardiograms were read centrally at the University of Pennsylvania by one reader who was blinded to the studies. 
LV mass was calculated using the area-length method and indexed to height ${ }^{2.7}$ [21]. LVH was defined as LV mass/height ${ }^{2.7}$ $\geq 47 \mathrm{~g} / \mathrm{m}^{2.7}$ in women and $\geq 50 \mathrm{~g} / \mathrm{m}^{2.7}$ in men $[22,23]$.

Relative wall thickness (RTW) was calculated as 2 times posterior wall thickness/LV internal linear dimension in diastole, and it was considered to be increased if $\geq 0.45$. LV mass and RTW were used to categorize LV geometry: normal (normal LV mass and normal RWT), concentric remodeling (normal LV mass and increased RWT), eccentric hypertrophy (increased LV mass and normal RWT), and concentric hypertrophy (increased LV mass and increased RWT).

LV systolic function was assessed using the EF obtained from echocardiograms. LV end-diastolic and end-systolic volumes were calculated using the modified biplane method. EF was calculated as (end-diastolic volume - end-systolic volume)/end-diastolic volume. LV systolic dysfunction was defined as EF <45\% [24].

LV diastolic function was categorized as normal or mildly, moderately, or severely abnormal (corresponding to normal and grades 1, 2, and 3 diastolic dysfunction) based on mitral inflow E- and A-wave velocities, E-wave deceleration time, and pulmonary venous reverse A-wave duration [25]. These parameters were unable to obtain in 564 participants due to equipment limitations of one CRIC center. A small subset $(<1 \%)$ of participants with atrial fibrillation could not have measurements of diastolic dysfunction.

\section{Covariates}

Demographic and clinical information were obtained at the baseline and follow-up study visits by questionnaires, interviews and physical examination. History of any cardiovascular disease (CVD) included prior myocardial infarction, revascularization, HF, stroke, or peripheral arterial disease. Current smoking was defined as self-report of current use of cigarettes and at least 100 cigarettes smoked. At each study visit, participants were queried about any medication usage in the prior 30 days. All anti-hypertensive medications were categorized into drug classes, and the total number of anti-hypertensive drug classes was calculated. All hospitalization records were reviewed and clinical events were adjudicated by 2 independent reviewers according to CRIC study protocol. eGFR was calculated using the CRIC study equation [26].

Diabetes mellitus was defined as a fasting glucose $>126 \mathrm{mg} / \mathrm{dl}$, a non-fasting glucose $>200 \mathrm{mg} / \mathrm{dl}$, or use of insulin or other antidiabetic medication [27]. Hypertension was defined as systolic blood pressure (BP) $\geq 140 \mathrm{~mm} \mathrm{Hg}$, diastolic $\mathrm{BP} \geq 90 \mathrm{~mm} \mathrm{Hg}$, or use of antihypertensive medications [28].

\section{Statistical Analysis}

The characteristics of study participants were depicted using standard descriptive statistics, overall and stratified by categories of serum bicarbonate $<22,22-26$ and $>26 \mathrm{mmol} / \mathrm{l}$. Pearson's $\chi^{2}$ for categorical variables and test for continuous variables were used to analyze the covariates of interest and their association with serum bicarbonate.

Multivariable linear regression models were used to assess the association between bicarbonate and LV mass, cross-sectional at CRIC study year 1. Secondary longitudinal analyses were performed during follow-up at CRIC study years 4 and 7. To address potential nonlinearity, restricted cubic spline models were created. The goodness of curve fit was assessed with the F test. Logistic re-

Serum Bicarbonate and LV Structure and Function gression models were built to assess the association between serum bicarbonate and LVH, LV systolic and diastolic dysfunction, crosssectional at study year 1 and during follow-up at years 4 and 7 . Diastolic dysfunction was dichotomized into normal vs. abnormal (corresponding to mildly, moderately and severely abnormal) relaxation. Multinomial logistic regression models were built to assess the 4 categories of LV geometry, using normal geometry as the reference group. The models were adjusted for relevant confounding variables, including demographics (age, gender, race/ethnicity), traditional CVD risk factors (hypertension, diabetes, smoking, low-density lipoprotein cholesterol, prior history of CVD), medications (angiotensin-converting enzyme inhibitors or angiotensin II receptor blockers, diuretics, anti-acidosis medications) and kidney function (eGFR and urine protein excretion). Sequential multivariable models for each outcome were created based on our assessment of the likelihood of covariates being a confounder in the relationship between serum bicarbonate and heart disease. All models were also adjusted for study clinical center to account for the potential variability across centers. Residual plots were used to confirm model assumptions.

The effect modification by an a priori selected set of baseline characteristics including race/ethnicity, diabetes status, diuretic use, level of kidney function (eGFR $<45$ and $\geq 45 \mathrm{ml} / \mathrm{min} / 1.73 \mathrm{~m}^{2}$ ) and proteinuria was also explored. Because the presence of CVD is correlated closely with recurrence of cardiovascular events, a secondary set of analyses was performed to evaluate the outcomes of interest in participants without known CVD. Additionally, we assessed the subsequent risk of serum bicarbonate to predict $\mathrm{LVH}$ in participants without known LVH at study year 1. Similarly, we evaluated the risk conferred by serum bicarbonate on the development of abnormal LV geometry during follow-up in participants with normal LV geometry at study year 1 . All statistical tests were 2 -sided, and $\mathrm{p}<0.05$ was considered significant. IBM Corp (2013) SPSS Statistics for Windows version 22.0, Armonk, N.Y., and R Development Core Team (2011). R: a language and environment for statistical computing, Vienna, Austria were used for analyses.

\section{Results}

The study cohort included 3,483 participants with a mean age of $58.9 \pm 10.8(\mathrm{SD})$, mean eGFR of $42.5 \mathrm{ml} /$ $\min / 1.73 \mathrm{~m}^{2}$, median 24 -hour urine total protein excretion of $0.2 \mathrm{~g} /$ day and median serum bicarbonate level of 24 ; interquartile range $22-26 \mathrm{mmol} / \mathrm{l}$. In the study cohort, 1,446 (41.5\%) were African Americans, 1,571 (45.1\%) were females and 1,726 (49.6\%) had diabetes. Compared with participants with serum bicarbonate $22-26 \mathrm{mmol} / \mathrm{l}$, participants in the low-bicarbonate group $(<22 \mathrm{mmol} / \mathrm{l})$ were more likely to be younger, Hispanic, with diabetes and hypertension and not on a diuretic. Additionally, they were more likely to have a lower eGFR, hemoglobin, and albumin and higher proteinuria, parathyroid hormone, phosphorus and fibroblast growth factor 23 levels (table 1). 
Table 1. Characteristics of CRIC study participants by groups of serum bicarbonate

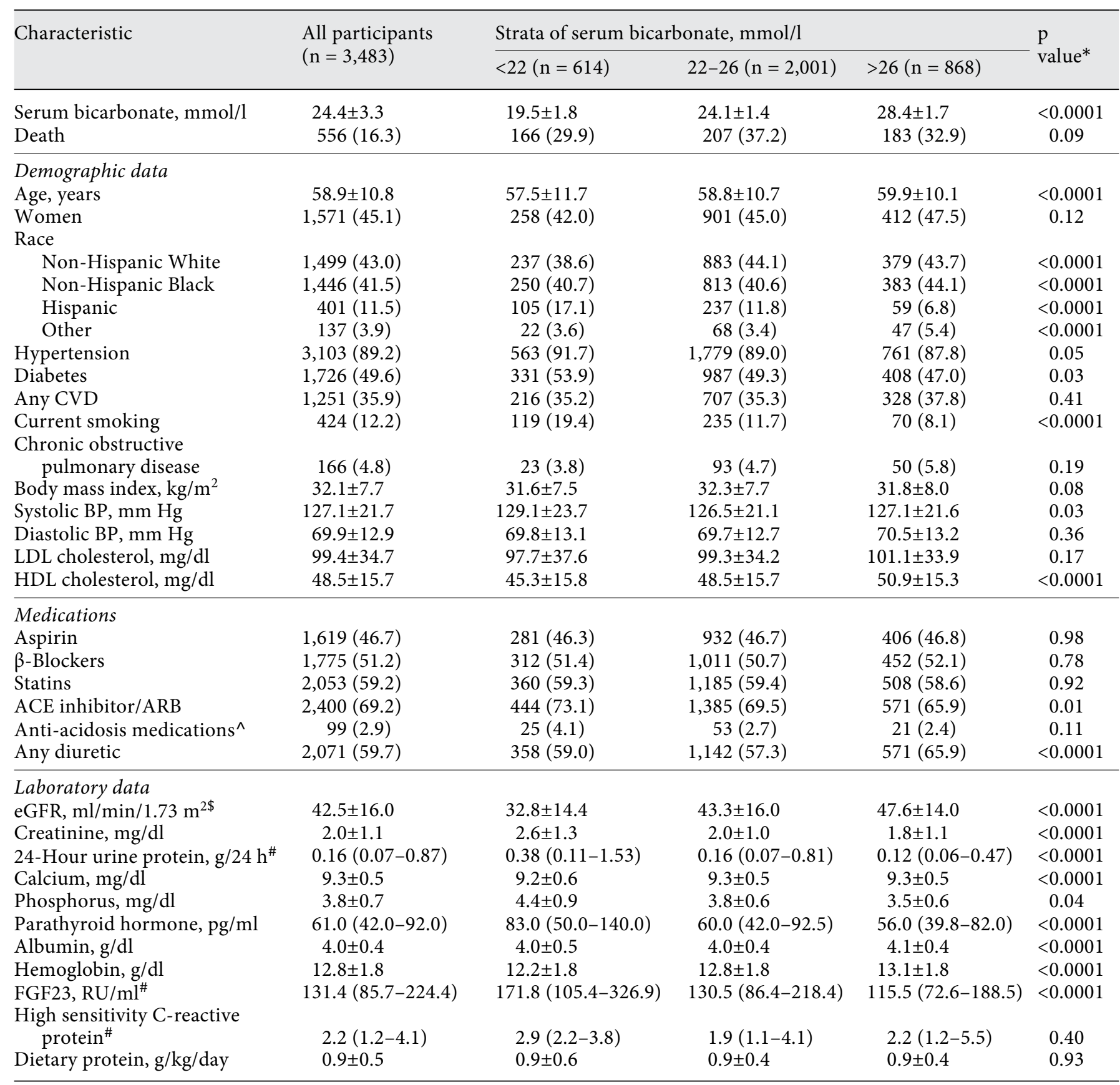

Unless otherwise noted, values are $\mathrm{n}(\%)$ or means \pm SDs.

$\mathrm{ACE}=$ Angiotensin-converting enzyme; $\mathrm{ARB}=$ angiotensin-receptor blocker; FGF23 = fibroblast growth factor 23 ; LDL = lowdensity lipoprotein; HDL = high-density lipoprotein.

* p value obtained from 1-way analysis of variance or chi-square as appropriate.

\# Median (interquartile range).

$\wedge$ Anti-acidosis medications are represented by: calcium citrate, magnesium citrate, potassium citrate, sodium bicarbonate, sodium lactate, sodium citrate, sodium, acetate, tromethamine, and lactated potassium saline.

\$ eGFR was calculated from serum creatinine and cystatin C using a CRIC study equation [47]. 


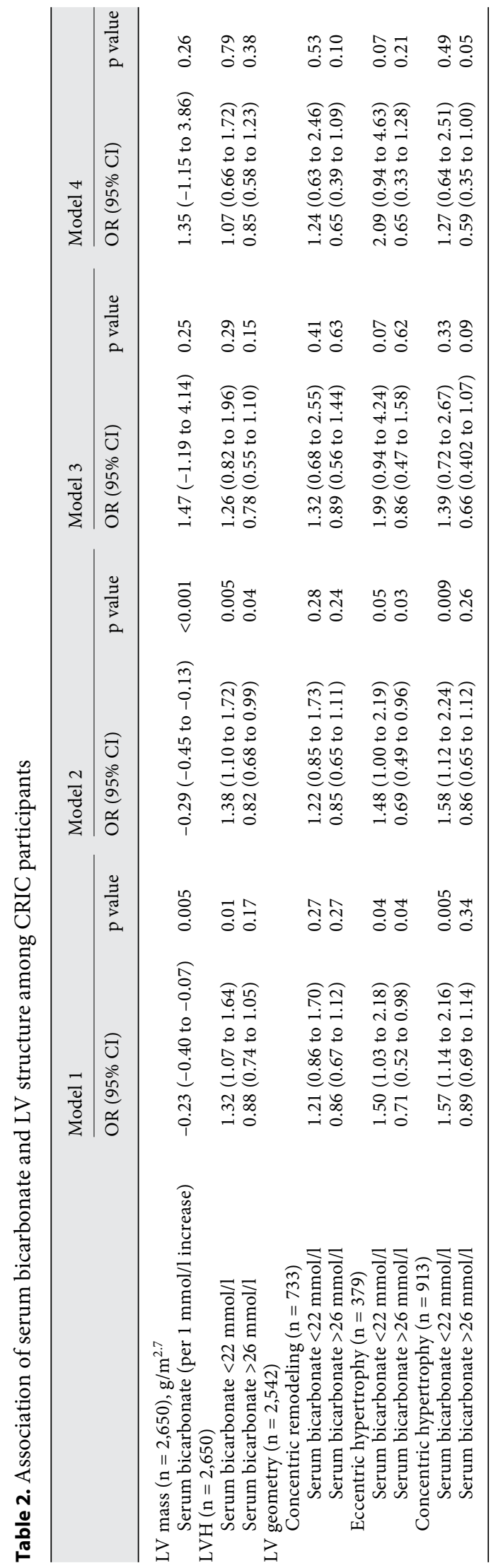

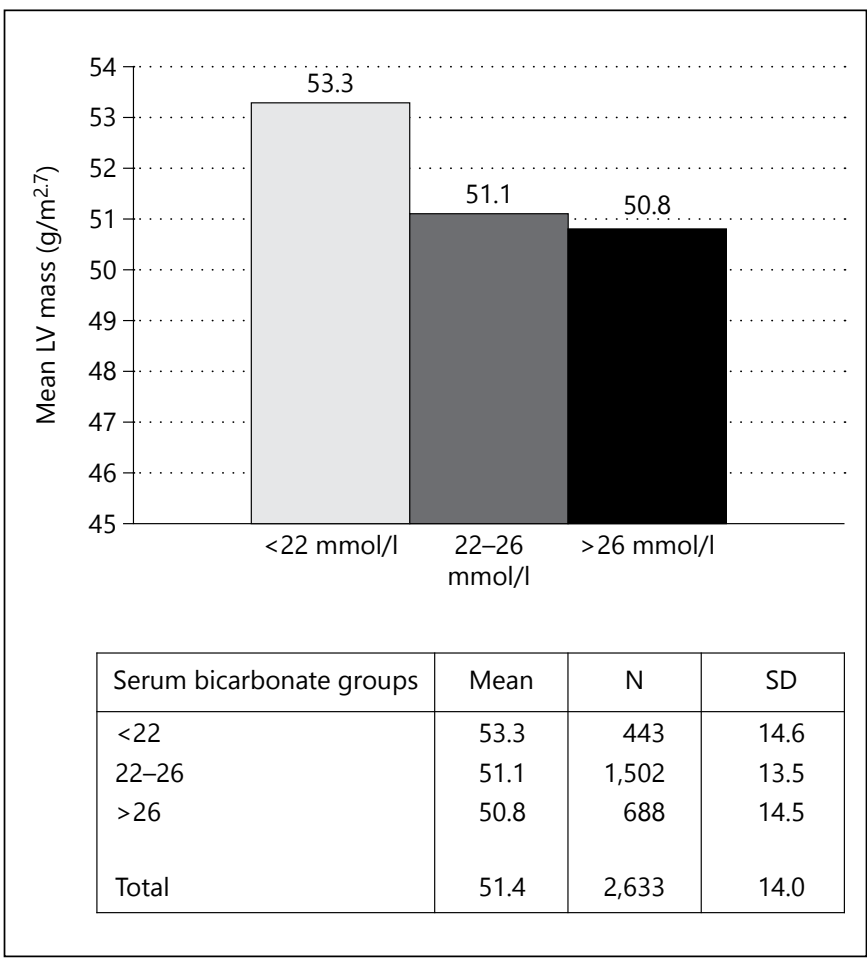

Fig. 1. Mean LV mass $\left(\mathrm{g} / \mathrm{m}^{2.7}\right)$ by serum bicarbonate categories.

\section{Serum Bicarbonate and Structural Cardiac Abnormalities}

$\mathrm{LV}$ mass was higher in participants in the stratum of bicarbonate $<22 \mathrm{mmol} / \mathrm{l}\left(53.3 \pm 14.6 \mathrm{~g} / \mathrm{m}^{2}\right)$ compared to stratum of bicarbonate $>26 \mathrm{mmol} / \mathrm{l}\left(50.5 \pm 14.5 \mathrm{~g} / \mathrm{m}^{2}, \mathrm{p}=\right.$ 0.002 ; fig. 1). In demographically adjusted models, there was an inverse association between bicarbonate and $\mathrm{LV}$ mass, with $0.29 \mathrm{~g} / \mathrm{m}^{2}$ lower LV mass with each $1 \mathrm{mmol} / 1$ increase in serum bicarbonate (OR $-0.29 ; 95 \% \mathrm{CI}-0.45$ to -0.13; table 2). After adjustments for traditional cardiovascular risk factors, the association lost its statistical significance (OR 1.47; 95\% CI -1.19 to 4.14 ). Additional adjustments for renal function and proteinuria did not further influence the association. Exploratory nonlinear modeling revealed lack of statistical significance for a nonlinear relationship between bicarbonate and LV mass ( $\mathrm{p}=0.13$; fig. 2).

The prevalence of LVH was $51.2 \%$ for the entire cohort, with 57.8, 50.5 and $47.7 \%$ for the low, normal and high serum bicarbonate groups respectively. In unadjusted and demographically adjusted models, associations of serum bicarbonate with LVH were significant, with nearly $40 \%$ higher risk of LVH for the low serum bicarbonate $(<22 \mathrm{mmol} / \mathrm{l})$ group (OR $1.38 ; 95 \% \mathrm{CI}$ $1.10-1.72$ ), and almost $20 \%$ lower risk of LVH for the 
high serum bicarbonate ( $>26 \mathrm{mmol} / \mathrm{l}$ ) group (OR 0.82; 95\% CI 0.68-0.99) when compared with the normal group (22-26 mmol/l; table 2). After multivariable adjustments, there was no statistically significant association between serum bicarbonate strata and LVH (table 2).

Approximately $80 \%$ of study participants had abnormal LV geometry: 28.9\% (729) concentric remodeling, $14.9 \%$ (377) eccentric hypertrophy and 35.8\% (905) concentric hypertrophy. The participants in the lowest bicarbonate stratum were more likely to have concentric hypertrophy $(40.8 \%, \mathrm{n}=173)$ compared to those in the normal $(34.7 \%, \mathrm{n}=501)$ and high-bicarbonate strata $(35.1 \%, \mathrm{n}=231), \chi=17.06, \mathrm{p}=0.009$. After adjustments for demographics, participants in the low-bicarbonate stratum $(<22 \mathrm{mmol} / \mathrm{l})$ were $58 \%$ more likely to have concentric hypertrophy, when compared to the group of bicarbonate 22-26 mmol/l (OR 1.58; 95\% CI 1.12-2.24, $\mathrm{p}=$ 0.009; table 2). Participants in the high-bicarbonate group were less likely to have abnormal LV geometry, and eccentric hypertrophy, in particular (OR 0.69; 95\% CI 0.49-0.96, $\mathrm{p}=0.03$ ). However, after multivariable adjustments, there was no statistically significant association between strata of serum bicarbonate and LV geometry (fig. 3).

\section{Serum Bicarbonate and Functional Cardiac \\ Abnormalities}

The majority of participants (88.6\%) had preserved EF $(\geq 45 \%)$. The odds of having systolic dysfunction (EF $<45 \%$ ) was similar across serum bicarbonate strata in univariate and multivariable adjusted models. There was no statistically significant association between bicarbonate levels and measures of diastolic dysfunction in multivariable logistic regression models, adjusted for demographics, traditional cardiovascular risk factors and kidney function (table 3).

\section{Longitudinal Changes in Structural and Functional \\ Cardiac Abnormalities}

Echocardiography measurements performed at study year 1 were repeated at subsequent study years 4 and 7 (table 4). Over time, no statistically significant changes were observed in LV mass or EF, in any of the bicarbonate strata. We examined the strength of association between serum bicarbonate at year 1 and progression to LVH in subsequent years, in the subgroup of participants without $\mathrm{LVH}$ at year 1 . Serum bicarbonate was not an independent predictor of development of LVH in this subgroup (online suppl. table 1S).

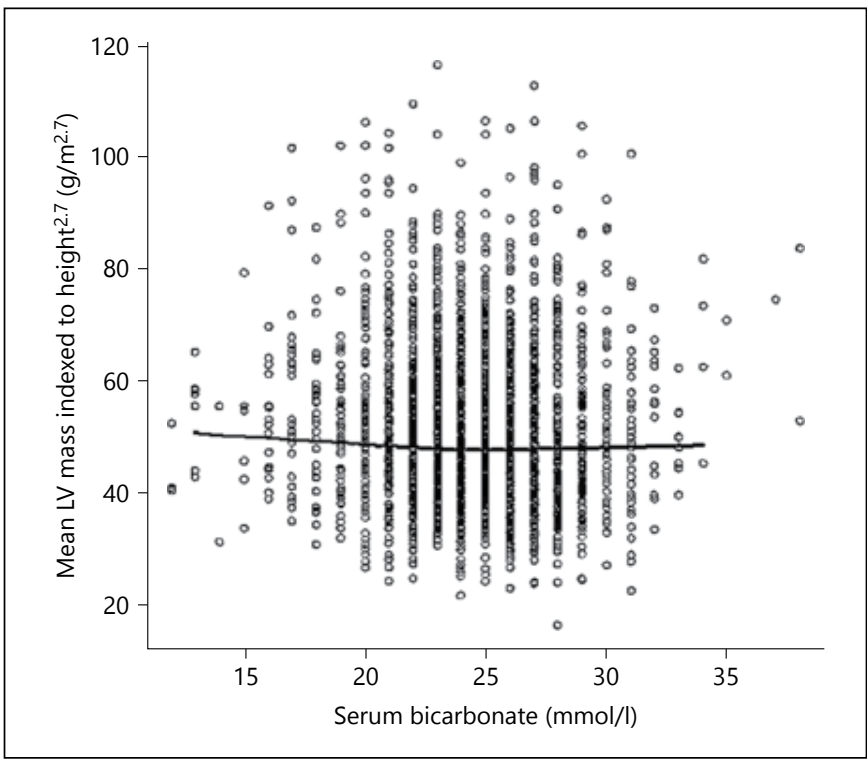

Fig. 2. Association between serum bicarbonate level ( $\mathrm{mmol} / \mathrm{l})$ and LV mass indexed to height at $2.7 \mathrm{~g} / \mathrm{m}^{2.7}$. Adjusted restricted cubic spline model with knots at 20,24 and 28. The solid line represents the effect $(\mathrm{p}=0.13)$.

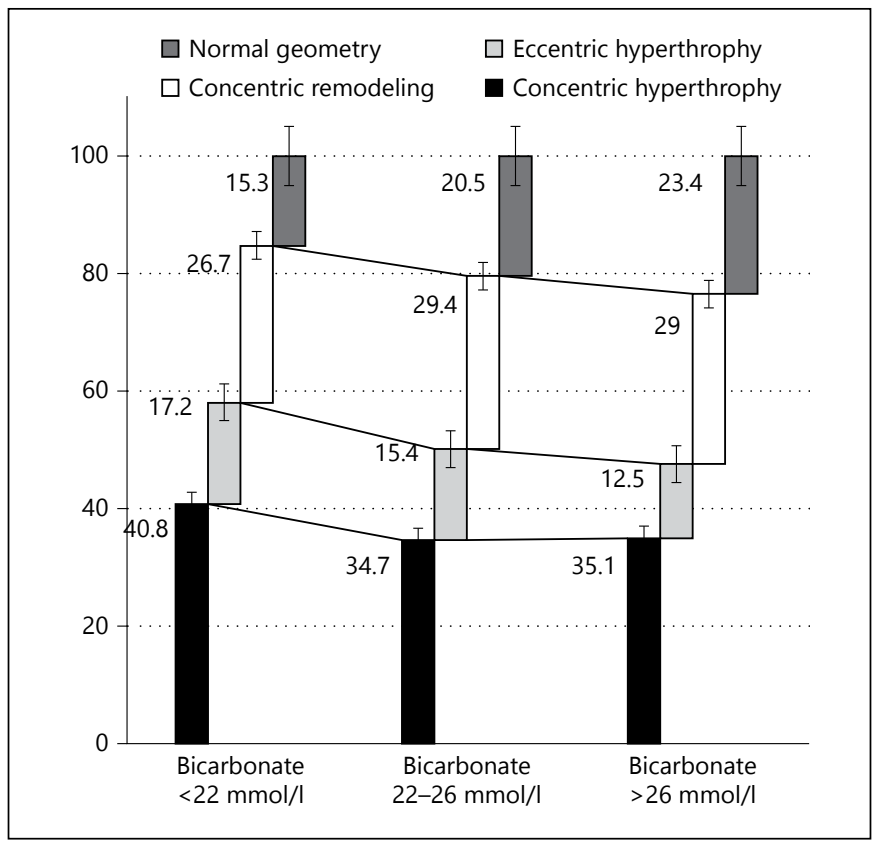

Fig. 3. LV geometry by the levels of serum bicarbonate.

Similarly, we explored the association between serum bicarbonate and LV geometry at study years 4 and 7, after exclusion of participants with abnormal geometry, with similar findings (online suppl. table $2 S$ ). 
Table 3. Association of serum bicarbonate and LV function among CRIC participants

\begin{tabular}{|c|c|c|c|c|}
\hline & \multicolumn{2}{|l|}{ Model 1} & \multicolumn{2}{|l|}{ Model 2} \\
\hline & OR (95\% CI) & $\mathrm{p}$ value & OR $(95 \% \mathrm{CI})$ & $\mathrm{p}$ value \\
\hline Serum bicarbonate $<22 \mathrm{mmol} / \mathrm{l}$ & $1.04(0.78-1.38)$ & 0.86 & $0.76(0.41-1.42)$ & 0.37 \\
\hline Serum bicarbonate $>26 \mathrm{mmol} / \mathrm{l}$ & $1.06(0.82-1.38)$ & 0.64 & $0.77(0.44-1.37)$ & 0.40 \\
\hline \multicolumn{5}{|l|}{ Diastolic dysfunction } \\
\hline Serum bicarbonate $>26 \mathrm{mmol} / \mathrm{l}$ & $0.99(0.81-1.20)$ & 0.88 & $1.03(0.83-1.28)$ & 0.80 \\
\hline \multicolumn{5}{|l|}{ Moderately abnormal relaxation } \\
\hline Serum bicarbonate $<22 \mathrm{mmol} / \mathrm{l}$ & $0.70(0.47-1.03)$ & 0.07 & $0.68(0.44-1.04)$ & 0.09 \\
\hline Serum bicarbonate $>26 \mathrm{mmol} / 1$ & $0.93(0.67-1.29)$ & 0.68 & $0.94(0.66-1.34)$ & 0.72 \\
\hline \multicolumn{5}{|l|}{ Severely abnormal relaxation } \\
\hline Serum bicarbonate $<22 \mathrm{mmol} / \mathrm{l}$ & $0.70(0.26-1.89)$ & 0.48 & $0.53(0.14-1.91)$ & 0.33 \\
\hline
\end{tabular}

$\mathrm{ACE}=$ Angiotensin-converting enzyme; $\mathrm{ARB}=$ angiotensin-receptor blocker; FGF23 = fibroblast growth factor 23; LDL = low-density lipoprotein.

Model 1 - unadjusted.

Model 2 - adjusted for demographics (age, gender, race) and clinical center, diabetes, hypertension, current tobacco use, CVD, diuretics, ACE inhibitor/ARB, anti-acidosis medication, LDL, FGF23, proteinuria and eGFR.

\section{Subgroup Analyses}

We performed a series of a priori defined subgroup analyses; the association between serum bicarbonate level and structural and functional cardiac abnormalities was consistent when stratified by race/ethnicity, diabetes status, proteinuria and eGFR levels (online suppl. table 3S). In other sensitivity analyses, we excluded participants with CVD at study baseline and/or in the first year, prior to first echocardiography evaluation; results were consistent (online suppl. table 4 S).

\section{Discussion}

CKD is frequently associated with HF and confers the highest risk of mortality and hospitalizations for HF [29]. Mechanisms underlying this association are incompletely understood. In this large cohort of individuals with mild to moderate CKD and without NYHA class III/IV $\mathrm{HF}$, we hypothesized that low serum bicarbonate, a feature of chronic metabolic acidosis observed in CKD, is associated with heart structural and functional abnormalities. We found that LV mass is higher, and LVH and abnormal LV geometry are more common in CKD patients with low serum bicarbonate. However, these associations were attenuated and not statistically significant after ad-
Table 4. Longitudinal changes in LV structure and function by serum bicarbonate groups

Serum bicarbonate groups at study year $1, \mathrm{mmol} / \mathrm{l}$

$<22 \quad 22-26 \quad>26$

LV mass indexed to height ${ }^{2.7 *}, \mathrm{~g} / \mathrm{m}^{2.7}$, mean $\pm \mathrm{SD}$

$\begin{array}{lrrr}\text { Year 1 } & 48.1 \pm 12.0 & 46.6 \pm 12.1 & 48.1 \pm 13.8 \\ \text { Year 4 } & 49.4 \pm 11.9 & 47.0 \pm 10.6 & 47.9 \pm 11.6 \\ \text { Year 7 } & 48.8 \pm 12.0 & 47.1 \pm 11.1 & 46.3 \pm 12.5 \\ \text { EF**, \%, mean } \pm \text { SD }^{*} & & \\ \text { Year 1 } & 55.2 \pm 6.9 & 54.3 \pm 7.5 & 54.9 \pm 7.9 \\ \text { Year 4 } & 51.9 \pm 8.4 & 51.7 \pm 8.2 & 51.6 \pm 8.5 \\ \text { Year 7 } & 48.3 \pm 8.7 & 48.7 \pm 8.5 & 49.0 \pm 8.8\end{array}$

* Analyses based on the same 448 CRIC participants with available measures of LV mass at study years 1, 4 and 7 .

** Analyses based on the same 597 CRIC participants with available measurements of LV EF at study years 1,4 and 7 .

justment for traditional cardiovascular risk factors and kidney function.

To our knowledge, this is the first study to comprehensively evaluate the association between serum bicarbonate and echocardiographic measures of cardiac structure and function in CKD patients. LV mass was higher, and LVH 
was more prevalent in participants in the lower serum bicarbonate stratum. Humoral regulatorymechanismsaimed to increase urinary acidification in CKD, including activation of angiotensin II, aldosterone and endothelin can cause direct cardiac damage [30-32]. The potential link between chronic acidosis in CKD and heart structure abnormalities could be mediated through inflammatory and neurohumoral mechanisms activation. Acidosis and inflammation are strongly linked in CKD [33-35]. It is reasonable to speculate that the chronic metabolic acidosis results in neurohormonal stimulation and increased inflammatory markers, which could contribute to changes in LV mass and geometry. However, in our study, adjustment for traditional risk factors for heart disease attenuated the association. This suggests that the association between serum bicarbonate and cardiac structure is confounded by the presence of common clinical conditions such as diabetes and hypertension, which may share similar inflammatory and neurohumoral milieu. It is also possible that the association between serum bicarbonate levels and clinical HF episodes is mediated by other factors such as changes in endothelial function and peripheral vascular resistance as discussed below but which are not captured by cardiac imaging.

Animal models described an association between acidosis and myocardial dysfunction. Acute acidemia causes depression of LV function in a dog model [14] and contributes to reduced myocardial contractility in rats with HF [36]. This can be physiologically explained. In order that crossbridges between actin and myosin be created and contraction may occur, the inhibitory action of troponin on actin-myosin interaction must be overcome. In the setting of high intracellular $\mathrm{H}^{+}$, a smaller percentage of the available calcium is able to react with troponin, fewer actin-myosin interactions occur, and the strength of contraction is reduced. Similarly, sarcoplasmic reticulum isolated from heart tissue releases less $\mathrm{Ca}$ when the $\mathrm{pH}$ of the medium is low. If the results are extrapolated to the intact animal, acute acidosis may cause a lower amount of calcium released during each beat and a subsequent diminished force of contraction [37].

Few studies have been done in humans to assess the effect of acidosis or alkalosis on the heart. Our previous study in CRIC [15] showed an increased risk of HF episodes both with high and low serum bicarbonate. Some of the symptoms of HF, including weakness and shortness of breath, can be caused by poor oxygen delivery to the peripheral tissues, an action affected by the blood $\mathrm{pH}$. It is reasonable to conceive that at any given increase in blood $\mathrm{pH}$, one would encounter reduced oxygen delivery that would clinically manifest as weakness, shortness of breath, fatigue, without any significant effect on heart structural or functional abnormalities. In human endstage failing myocardium, mild acute acidosis impaired contractility and the b-adrenergic response [38]. However, other studies failed to show a link between acidosis and impaired cardiac function [39] and that debate was solved with the discovery that the direct effect of acute acidosis on the myocardium could be masked by the effect of catecholamines $[40,41]$. This might be indeed the rationale behind our previous found link between serum bicarbonate and HF events, but negative association with systolic or diastolic dysfunction. Neurohumoral activation of sympathetic and renin-angiotensin-aldosterone systems induced by chronic acidosis potentially leads to endothelial dysfunction, increase in peripheral venous and arterial resistance, with subsequent increase in preload and afterload, progressive retention of salt and water and edema. However, this hypothesis cannot be directly tested in our current analyses. In addition, the lack of association of serum bicarbonate with systolic dysfunction might not be unexpected, given that patients with NYHA III/IV were excluded from study enrollment and only about $11 \%$ had a low EF at study year 1 .

This study has the following key strengths: it represents the largest and most comprehensive analyses of the association between serum bicarbonate and cardiac structure and function in CKD. The large and racially diverse patient population, long duration of follow-up, comprehensive covariate measurements, and large subgroup sizes that allow robust subgroup analyses are also important strengths. However, there are important limitations too. Cardiac structure and function measurements were done using 2D mode echocardiography and not cardiac magnetic resonance. LV mass evaluated by cardiac magnetic resonance imaging is more precise and reliable compared to that provided by the $2 \mathrm{D}$ mode echocardiography $[42,43]$. Furthermore, the ability of 2D mode echocardiography technique to detect serial changes in LV mass measurements may be limited $[44,45]$. Therefore, it is possible that the lack of statistical significance in our models may be due to echocardiograms measurement bias. Second, serum bicarbonate alone is not a true estimation of the acid base status, but blood gas analyses were not available in CRIC. We did however adjust the analyses for the presence of lung disease, since some participants might have high bicarbonate as a compensatory mechanism for respiratory acidosis. Third, serum bicarbonate measurements occurred 1-2 days after the original blood draw, and there is a reduction of up to $4 \mathrm{mmol} / \mathrm{l}$ in serum bicarbonate concentration for all determinations done 24-48 h from collection; however, this time gap in 
measurement is common in large epidemiologic studies that use a central laboratory [46]. Lastly, data on valve replacement or cardiac resynchronization therapies that could potentially influence cardiac structure and function were not available; this limited the interpretation of the longitudinal changes in cardiac structure, but not the detailed cross-sectional analyses reported in this paper.

In summary, in this large cohort of patients with CKD, serum bicarbonate was not independently associated with cardiac structural and functional abnormalities. These findings suggest that the association between serum bicarbonate and heart disease are either confounded or mediated by other more traditional cardiovascular risk factors. Other perhaps neurohumoral mechanisms, activated by changes in acid base balance may mediate the association between serum bicarbonate and HF events and merit further studies.

\section{Sources of Funding}

Funding for the CRIC study was obtained under a cooperative agreement from NIDDK (U01DK060990, U01DK060984, U01DK061022, U01DK061021, U01DK061028, U01DK060980,
U01DK060963, and U01DK060902). In addition, this work was supported in part by: University of Pennsylvania Clinical and Translational Science Award National Institutes of Health (NIH)/National Center for Advancing Translational Sciences (NCATS) UL1TR000003, Johns Hopkins University UL1 TR000424, University of Maryland GCRC M01 RR-16500, Clinical and Translational Science Collaborative of Cleveland, UL1TR000439 from the NCATS component of the NIH and NIH roadmap for Medical Research, Michigan Institute for Clinical and Health Research UL1TR000433, University of Illinois at Chicago CTSA UL1RR029879, Tulane University Translational Research in Hypertension and Renal Biology P30GM103337, Kaiser Permanente NIH/NCRR UCSF-CTSI UL1 RR-024131. M.D. is supported by 13FTF15920005. K.S. is supported by DK094352-01.

\section{Disclosure Statement}

The authors have no conflict of interest to disclose.

\section{Appendix}

Alan S. Go, MD, Jiang He, MD, John W. Kusek, PhD, Akinlolu Ojo, MD, James P. Lash, MD, Raymond R. Townsend, MD.

\section{References}

1 Kraut JA, Kurtz I: Metabolic acidosis of CKD: diagnosis, clinical characteristics, and treatment. Am J Kidney Dis 2005;45:978-993.

2 Bailey JL: Metabolic acidosis: an unrecognized cause of morbidity in the patient with chronic kidney disease. Kidney Int Suppl 2005;96:S15-S23.

3 Schwartz WB, Hall PW 3rd, Hays RM, Relman AS: On the mechanism of acidosis in chronic renal disease. J Clin Invest 1959;38(1, pt 1):39-52.

4 Relman AS: Renal acidosis and renal excretion of acid in health and disease. Adv Intern Med 1964;12:295-347.

5 Herzog CA, Asinger RW, Berger AK, Charytan DM, Díez J, Hart RG, Eckardt KU, Kasiske BL, McCullough PA, Passman RS, DeLoach SS, Pun PH, Ritz E: Cardiovascular disease in chronic kidney disease. A clinical update from kidney disease: improving global outcomes (KDIGO). Kidney Int 2011;80:572586.

6 Glassock RJ, Pecoits-Filho R, Barberato SH: Left ventricular mass in chronic kidney disease and ESRD. Clin J Am Soc Nephrol 2009; 4(suppl 1):S79-S91.

7 Park M, Hsu CY, Li Y, Mishra RK, Keane M, Rosas SE, Dries D, Xie D, Chen J, He J, Anderson A, Go AS, Shlipak MG: Associations between kidney function and subclinical cardiac abnormalities in CKD. J Am Soc Nephrol 2012;23:1725-1734.

8 Levin A, Thompson CR, Ethier J, Carlisle EJ, Tobe S, Mendelssohn D, Burgess E, Jindal K, Barrett B, Singer J, Djurdjev O: Left ventricular mass index increase in early renal disease: impact of decline in hemoglobin. Am J Kidney Dis 1999;34:125-134.

9 Eckardt KU, Scherhag A, Macdougall IC, Tsakiris D, Clyne N, Locatelli F, Zaug MF, Burger HU, Drueke TB: Left ventricular geometry predicts cardiovascular outcomes associated with anemia correction in CKD. J Am Soc Nephrol 2009;20:2651-2660.

10 Payne J, Sharma S, De Leon D, Lu JL, Alemu F, Balogun RA, Malakauskas SM, KalantarZadeh K, Kovesdy CP: Association of echocardiographic abnormalities with mortality in men with non-dialysis-dependent chronic kidney disease. Nephrol Dial Transplant 2012;27:694-700.

11 Martin FL, McKie PM, Cataliotti A, Sangaralingham SJ, Korinek J, Huntley BK, Oehler EA, Harders GE, Ichiki T, Mangiafico S, Nath KA, Redfield MM, Chen HH, Burnett JC Jr: Experimental mild renal insufficiency mediates early cardiac apoptosis, fibrosis, and diastolic dysfunction: a kidney-heart connection. Am J Physiol Regul Integr Comp Physiol 2012; 302:R292-R299.
12 Khabbaz KR, Feng J, Boodhwani M, Clements RT, Bianchi C, Sellke FW: Nonischemic myocardial acidosis adversely affects microvascular and myocardial function and triggers apoptosis during cardioplegia. J Thorac Cardiovasc Surg 2008;135:139-146.

13 Brown RH Jr, Cohen I, Noble D: The interactions of protons, calcium and potassium ions on cardiac Purkinje fibres. J Physiol 1978;282: 345-352.

14 Mitchell JH, Wildenthal K, Johnson RL Jr: The effects of acid-base disturbances on cardiovascular and pulmonary function. Kidney Int 1972;1:375-389.

15 Dobre M, Yang W, Chen J, et al: Association of serum bicarbonate with risk of renal and cardiovascular outcomes in CKD: a report from the chronic renal insufficiency cohort (CRIC) study. Am J Kidney Dis 2013;62:670678.

16 Dobre M, Yang W, Pan Q, et al: Persistent high serum bicarbonate and the risk of heart failure in patients with chronic kidney disease (CKD): a report from the chronic renal insufficiency cohort (CRIC) study. J Am Heart Assoc 2015;4:pii:e001599.

17 Nassif ME, Novak E, Rich MW: Association of serum bicarbonate with long-term outcomes in patients hospitalized with heart failure. Int J Cardiol 2014;177:673-675. 
18 Feldman HI, Appel LJ, Chertow GM, et al: The chronic renal insufficiency cohort (CRIC) study: design and methods. J Am Soc Nephrol 2003;14:S148-S153.

19 Lash JP, Go AS, Appel LJ, et al: Chronic renal insufficiency cohort (CRIC) study: baseline characteristics and associations with kidney function. Clin J Am Soc Nephrol 2009;4: 1302-1311.

20 Fischer MJ, Go AS, Lora CM, Ackerson L, Cohan J, Kusek JW, Mercado A, Ojo A, Ricardo AC, Rosen LK, Tao K, Xie D, Feldman HI, Lash JP; CRIC and H-CRIC Study Groups: CKD in Hispanics: baseline characteristics from the CRIC (chronic renal insufficiency cohort) and Hispanic-CRIC studies. Am J Kidney Dis 2011;58:214-227.

21 Lang RM, Bierig M, Devereux RB, et al: Recommendations for chamber quantification: a report from the American society of echocardiography's guidelines and standards committee and the chamber quantification writing group. J Am Soc Echocardiogr 2005;18: 1440-1463.

22 de Simone G, Devereux RB, Daniels SR, Koren MJ, Meyer RA, Laragh JH: Effect of growth on variability of left ventricular mass: assessment of allometric signals in adults and children and their capacity to predict cardiovascular risk. J Am Coll Cardiol 1995;25: 1056-1062.

23 Lauer MS, Anderson KM, Kannel WB, Levy D: The impact of obesity on left ventricular mass and geometry. The Framingham heart study. JAMA 1991;266:231-236.

24 Massie BM, Carson PE, McMurray JJ, et al: Irbesartan in patients with heart failure and preserved ejection fraction. N Engl J Med 2008;359:2456-2467.

25 Nagueh SF, Appleton CP, Gillebert TC, Marino $\mathrm{PN}, \mathrm{Oh} \mathrm{JK}$, Smiseth OA, Waggoner AD, Flachskampf FA, Pellikka PA, Evangelista A: Recommendations for the evaluation of left ventricular diastolic function by echocardiography. J Am Soc Echocardiogr 2009;22:107133.

26 Anderson AH, Yang W, Hsu CY, Joffe MM, Leonard MB, Xie D, Chen J, Greene T, Jaar BG, Kao P, Kusek JW, Landis JR, Lash JP, Townsend RR, Weir MR, Feldman HI; CRIC Study Investigators: Estimating GFR among participants in the chronic renal insufficiency cohort (CRIC) study. Am J Kidney Dis 2012; 60:250-261.
27 American Diabetes Association: Diagnosis and classification of diabetes mellitus. Diabetes Care 2006;29(suppl 1):S43-S48.

28 Chobanian AV, Bakris GL, Black HR, et al: The seventh report of the joint national committee on prevention, detection, evaluation, and treatment of high blood pressure: the JNC 7 report. JAMA 2003;289:2560-2572.

29 van Deursen VM, Urso R, Laroche C, Damman K, Dahlström U, Tavazzi L, Maggioni AP, Voors AA: Co-morbidities in patients with heart failure: an analysis of the European heart failure pilot survey. Eur J Heart Fail 2014;16:103-111.

30 Sayed-Ahmed MM, Khattab MM, Gad MZ, Osman AM: Increased plasma endothelin-1 and cardiac nitric oxide during doxorubicininduced cardiomyopathy. Pharmacol Toxicol 2001;89:140-144.

31 Sikkeland LI, Dahl CP, Ueland T, Andreassen AK, Gude E, Edvardsen T, Holm T, Yndestad A, Gullestad L, Kongerud J, Aukrust P, Øie E: Increased levels of inflammatory cytokines and endothelin-1 in alveolar macrophages from patients with chronic heart failure. PLoS One 2012;7:e36815.

32 Wesson DE, Simoni J, Broglio K, Sheather S: Acid retention accompanies reduced GFR in humans and increases plasma levels of endothelin and aldosterone. Am J Physiol Renal Physiol 2011;300:F830-F837.

33 Kalantar-Zadeh K, Mehrotra R, Fouque D, Kopple JD: Metabolic acidosis and malnutrition-inflammation complex syndrome in chronic renal failure. Semin Dial 2004;17: 455-465.

34 Rao M, Wong C, Kanetsky P, Girndt M, Stenvinkel P, Reilly M, Raj DS: Cytokine gene polymorphism and progression of renal and cardiovascular diseases. Kidney Int 2007;72: 549-556.

35 Recio-Mayoral A, Banerjee D, Streather C, Kaski JC: Endothelial dysfunction, inflammation and atherosclerosis in chronic kidney disease - a cross-sectional study of predialysis, dialysis and kidney-transplantation patients. Atherosclerosis 2011;216:446-451.

36 Kemi OJ, Arbo I, Høydal MA, Loennechen JP, Wisløff U, Smith GL, Ellingsen Ø: Reduced $\mathrm{pH}$ and contractility in failing rat cardiomyocytes. Acta Physiol (Oxf) 2006;188:185-193.

37 Nakamaru Y, Schwartz A: Possible control of intracellular calcium metabolism by $[\mathrm{H}+]$ : sarcoplasmic reticulum of skeletal and cardiac muscle. Biochem Biophys Res Commun 1970;41:830-836
38 Schotola H, Toischer K, Popov AF, Renner A, Schmitto JD, Gummert J, Quintel M, Bauer M, Maier LS, Sossalla S: Mild metabolic acidosis impairs the $\beta$-adrenergic response in isolated human failing myocardium. Crit Care 2012;16:R153.

39 Schutte E, Lambers Heerspink HJ, Lutgers HL, Bakker SJ, Vart P, Wolffenbuttel $\mathrm{BH}$, Umanath K, Lewis JB, de Zeeuw D, Gansevoort RT: Serum bicarbonate and kidney disease progression and cardiovascular outcome in patients with diabetic nephropathy: a post hoc analysis of the RENAAL (reduction of end points in non-insulin-dependent diabetes with the angiotensin II antagonist losar$\tan$ ) study and IDNT (irbesartan diabetic nephropathy trial). Am J Kidney Dis 2015;66: 450-458.

40 Smith NY, Corbascio AN: Myocardial resistance to metabolic acidosis. Arch Surg 1966; 92:892-897.

41 Rocamora JM, Downing SE: Preservation of ventricular function by adrenergic influences during metabolic acidosis in the cat. Circ Res 1969;24:373-381.

42 Bottini PB, Carr AA, Prisant LM, Flickinger FW, Allison JD, Gottdiener JS: Magnetic resonance imaging compared to echocardiography to assess left ventricular mass in the hypertensive patient. Am J Hypertens 1995;8: 221-228.

43 Grothues F, Smith GC, Moon JC, Bellenger NG, Collins P, Klein HU, Pennell DJ: Comparison of interstudy reproducibility of cardiovascular magnetic resonance with two-dimensional echocardiography in normal subjects and in patients with heart failure or left ventricular hypertrophy. Am J Cardiol 2002; 90:29-34.

44 Stuckey DJ, Carr CA, Tyler DJ, Clarke K: Cine-MRI versus two-dimensional echocardiography to measure in vivo left ventricular function in rat heart. NMR Biomed 2008;21: 765-772.

45 Rajappan K, Bellenger NG, Melina G, Di Terlizzi M, Yacoub MH, Sheridan DJ, Pennell DJ: Assessment of left ventricular mass regression after aortic valve replacement - cardiovascular magnetic resonance versus M-mode echocardiography. Eur J Cardiothorac Surg 2003; 24:59-65.

46 Boyanton BL Jr, Blick KE: Stability studies of twenty-four analytes in human plasma and serum. Clin Chem 2002;48:2242-2247. 